\title{
Chronique d'une réforme foncière dans la trajectoire politique du Mali
}

\section{Monique Bertrand}

\section{(2) OpenEdition}

1 Journals

Édition électronique

URL : http://journals.openedition.org/anthropodev/698

DOI : 10.4000/anthropodev.698

ISSN : 2553-1719

Éditeur

APAD - Association pour l'anthropologie du changement social et du développement

\section{Édition imprimée}

Date de publication : 1 décembre 2018

Pagination : 141-168

ISBN : 979-10-93476-06-3

ISSN : 2276-2019

\section{Référence électronique}

Monique Bertrand, «Chronique d'une réforme foncière dans la trajectoire politique du Mali »,

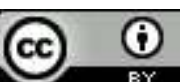

La revue Anthropologie \& développement est mise à disposition selon les termes de la Licence Creative Commons Attribution 4.0 International. 


\title{
Chronique d'une réforme foncière dans la trajectoire politique du Mali
}

\author{
Monique Bertrand
}

La réforme de la gestion domaniale et foncière lancée au Mali en 2014 fait suite à la crise qui vient de secouer le pays tout en renouant avec des révisions juridiques quasi-décennales sur ce sujet. La restauration du crédit de l'État est en jeu, mais elle maintient une référence ambiguë au régime qui est précisément tombé en 2012 : à la fois modèle d'expertise à suivre pour que convergent, autour du Cadastre, la nécessaire révision de la gestion publique et la résolution des problèmes fonciers, et exemple repoussoir de spéculation et de prédation dont l'administration malienne est complice. Ce texte interroge les conditions de mise en agenda politique de la réforme durant le mandat "post-crise ". Si son impulsion et ses financements sont liés à une plus générale circulation de modèles internationaux, il convient de les relier aussi aux contraintes endogènes de légitimation et de traduction de l'action. Trois niveaux d'intervention sont envisagés - format institutionnel, style ministériel, ordinaire bureaucratique -, chacun avec sa ligne de bénéfice politique recherché.

The reform of land management launched in 2014 in Mali comes after the crisis that just shook the country, and revives with legal revisions brought every ten-year to the land issue. The restoration of the State political credit is at stake, but its reference to the previous regime, which fell precisely in 2012, is ambiguous. At the same time: a model to be followed for arguing the Cadastre as the best expertise-based solution vis-à-vis the land concerns and the need for a new public management; a repulsive example of speculation and predation that the Malian administration is complicit in. This paper examines the way the reform has been set into a political agenda during the "post-crisis" mandate. If its impetus and financing are obviously influenced by external models circulating at a global scale, it also brings into play the endogenous constraints of legitimation and implementation faced by the Malian State. Three levels of action are considered - the institutional interplay, the minister's 
personal involvement, the ordinary work lead in land offices -, each of them looking for political benefits.

\section{Introduction}

Le 16 octobre 2014, une table ronde rassemblant à Bamako plus d'une centaine de participants, des représentants du gouvernement et des partenaires étrangers, a officiellement lancé la « réforme de la gestion foncière visant l'instauration du cadastre au Mali ». La rencontre donne l'occasion d'apprécier les termes de référence susceptibles de rallier les bailleurs à une priorité d'action publique. Elle interroge aussi les conditions de mise en agenda politique de mesures présentées comme constitutives d'une réforme. En désigner l'extraversion n'a d'ailleurs d'intérêt que si ces sources d'impulsion et de financement, dans une plus générale circulation de modèles internationaux, sont reliées aux contraintes endogènes de légitimation et de traduction de l'action. Ce texte les envisage selon leur propre temporalité. Pour ce faire, il déroule trois lignes de bénéfice politique recherché dans ce qui est plus une remise à l'agenda qu'une première mise en mots de la sécurisation foncière.

Le premier niveau de lecture est en effet celui des politiques publiques ou de gestions tenant lieu de policies. Dans un pays marqué par l'héritage juridique français, le rôle de l'État sur les terres du Domaine national confère à son administration de fortes prérogatives en matière de cession foncière ; la délivrance de titres définitifs de propriété lui revient à travers la tenue du Livre foncier qui enregistre ces droits formels. À l'heure du libéralisme et de la bonne gouvernance, l'action publique trouve ainsi son premier argument dans la nécessaire relecture du corpus juridique relatif à cette autorité concédante. Mais après les deux précédentes refontes de 1987 et 2000, la dernière reconnaissant enfin les droits des propriétaires coutumiers sur le Domaine de l'État, ce Code domanial et foncier n'a pas rendu le droit plus inclusif, et n'a pas réussi à débarrasser la pratique administrative d'une part d'informalité.

Un deuxième intérêt à suivre la réforme des années 2010 relève de l'analyse des politics. II s'agit de suivre des enjeux de captation et d'instrumentalisation politique de la rente foncière, d'arbitrage des concurrences à l'œuvre autour de différentes compétences administratives 
et des groupes sociaux qui en usent. L'intérêt économique suscité par les titres définitifs depuis les années 2000 bouscule notamment les régimes subalternes des coutumes et de la concession précaire, laquelle est rebaptisée "titre provisoire". Le portage politique de la réforme offre donc une clef de lecture des jeux de pouvoir à l'œuvre dans l'État et dans son fonctionnement en silos. La ressource foncière suscite en effet de constantes tensions entre des bureaucraties jalouses de leur information, peu disposées à la concertation, promptes à déclarer les autres incompétentes pour justifier leurs propres raccourcis de procédure. Sous ces formes fragmentées et clientélistes, la cession de droits formels de propriété entretient un ensemble de plaintes désignant tous les niveaux de l'État.

Moins de 15 ans après la rédaction du dernier Code, le lancement de la réforme suscite enfin un troisième niveau de mesure de la vie institutionnelle malienne, celui de l'entrepreneuriat toujours personnalisé qui tient lieu de volonté politique. Incarnant un récit national et ses normes morales, ceux que l'opinion désigne comme " politiciens avisés ", "grands républicains " ou "personnalités remarquables » de la société civile, inscrivent le pouvoir à l'intersection de communications intérieures et extérieures. Sans eux, ni plaidoyer des attentes sociales, ni promesse d'évaluation de la réforme; ni acceptation par l'usager de base du service domanial, ni lueur d'espoir donné aux victimes de spoliations foncières. Cette importance des positionnements personnels au sommet de l'État fait de nouveau le lien avec de plus modestes médiations culturelles à sa base administrative (Darbon, 2002).

Le pari de sécuriser droits et investissements fonciers n'a pourtant rien d'original au Mali ; la publicisation du débat sous la forme d'une table ronde n'est pas davantage inédite; la puissance publique conserve un rôle central de gestion, et les fortes pressions sociales auxquelles elle s'expose maintiennent une incapacité chronique à maîtriser le foncier. Mais la réforme intervient dans un moment singulier de l'histoire du pays, en réponse au putsch militaire et à l'occupation terroriste qu'il a connus en 2012. Après les élections présidentielles et législatives d'août-novembre 2013, elle s'insère dans un contexte présenté comme de "sortie de crise ": non pas dans un cadre routinier de renforcement de capacités institutionnelles, comme le pays en a connu pendant deux décennies en 
profitant de la manne internationale (Konaté, 2013), mais en opération de sauvetage de la cohésion sociale et du crédit territorial de l'État.

Dans ce moment dilaté sur quelques années, le montage et les premières mesures du chantier permettent d'envisager le condensé d'enjeux que porte ce triple fil politique : d'une commande présidentielle à son maître d'œuvre, le ministère des Domaines de l'État et des Affaires foncières (MDEAF); d'une parole adressée aux " citoyens démunis " aux communications à Paris et Washington ${ }^{1}$; de la perspective d'un data center national aux demandes de titres traitées dans des bureaux domaniaux locaux. L'analyse est menée ici depuis un environnement urbain (Bertrand, 2019); mais elle rend compte plus banalement des tensions que connaît l'action publique quand celle-ci est à la fois source des problèmes et porteuse des solutions. Le dossier du foncier bouscule ainsi la vie institutionnelle tout en s'accommodant de ses limites: en réargumentant des préparatifs, en retoquant des urgences, en rabotant la mise en œuvre. Son issue provisoire, à l'horizon 2018, ne surprend pas non plus dans un pays qui reste embourbé dans une crise multidimensionnelle.

\section{3-2014 : assainir la gouvernance du foncier, ça commence quand?}

La table ronde d'octobre 2014 cherche d'abord à démontrer que le Mali a repris un cours politique normal et le train des réformes. Elle aborde le sujet sous l'angle de la dématérialisation de l'information foncière: numérisation des dossiers établis pour les titres, migration des inventaires de droits existants vers de nouvelles bases de données, précision accrue des GPS indiquant les coordonnées géographiques des parcelles... II s'agit de convaincre les bailleurs du pays, à peine de retour et toujours inquiets d'une paix qui tarde à revenir au Nord, que la réforme sera érigée en priorité gouvernementale à inscrire dans l'aide budgétaire directe du pays.

\footnotetext{
${ }^{1}$ La France est le chef de file des partenaires du Mali lors de la table ronde, après avoir mis un conseiller technique à disposition du MDEAF ; le ministre malien intervient à trois reprises à la conférence Land and Poverty de la Banque mondiale.
} 


\section{Vers un discours de la méthode?}

À ce prisme du "toutes les parties prenantes", une approche narrative des politiques foncières s'impose (Zittoun, 2013). Celle-ci ne se limite pas à la reprise d'éléments de langage produits par les " partenaires techniques et financiers " (PTF), après évaluation mitigée, constat du gâchis ou d'échec ressortant de précédents "projets" de renforcement des capacités institutionnelles du pays. Bien des agendas de développement ont déjà montré comment les urgences produites par l'aide extérieure se voient converties par les élites nationales en élément de domination. II s'agit de considérer comment la fabrique discursive de la réforme interne constitue aussi une opération interne d' "intéressement" de divers mondes sociaux et de réenchantement de la sphère politique.

L'électorat malien se montre en effet sensible aux énoncés désignant les missions protectrices de l'État. Le lexique de la sécurisation des droits fonciers s'articule ainsi avec celui de la remise en ordre du pays. II fonctionne comme antidote d'un sentiment diffus de vulnérabilité qui dans les années 2010 relie des risques environnementaux et financiers, les violences physiques subies au Nord et la violence économique vécue au Sud. Des syndicats, les professions libérales désormais représentées en ordres professionnels, les représentants de la diaspora, les classes moyennes salariées, dont le logement est repoussé en périphérie urbaine dans un désert de services essentiels..., tous relaient ce sentiment d'insécurité (Sow, 2016). Le Cadastre - avec majuscule - est vu alors comme l'occasion de moderniser le pays, et par-delà l'angle d'attaque d'un continuum de sécurités à assurer sur les plans foncier et militaire, routier et alimentaire, sanitaire et énergétique... On en vient ainsi à repositionner le Maliba - un grand territoire malien, valant grandeur du Mali - dans la sous-région, et à pousser la fierté nationale vers plus de cohésion politique.

En effet, la crise politique de 2012 a été fortement ressentie dans le pays. L'action publique se réengage alors dans les termes du sursaut contre le délitement de la décennie précédente et contre le discrédit de la démocratie malienne. De ce fait, la réforme en préparation entretient une relation ambiguë avec le contexte d'avant-crise, à la fois repoussoir et référentiel d'action. La gouvernance de "consensus" portée par le 
président Amadou Toumani Touré se serait dévoyée (Brunet-Jailly et al., 2014) ; une fois celui-ci balayé, il convient de redorer le blason de la chose publique. La gestion domaniale des années 2000 est donc rappelée sous deux angles opposés.

Le premier est celui de la " poudrière " et du « brûlot » fonciers qui ont précipité la crise politique en généralisant " magouilles et confusions " au cœur de l'État. Les alertes et les dénonciations se sont multipliées sous la plume de hauts cadres, dans la presse, dans les manifestations de victimes (Bertrand, 2014). Les " affaires de terrains " montrent la cohésion sociale sous un jour critique; elles exposent publiquement la corruption municipale, la prévarication administrative et l'impunité qui résument la crise de gouvernance.

Le second angle est celui de la source d'inspiration, car le précédent président a fait émerger plusieurs scènes de dialogue dédiées aux problèmes fonciers. Parmi ces occasions de rencontre des institutions et de la société civile, les "États Généraux sur le foncier » tenus en 2009 ont multiplié les diagnostics et les signes de mobilisation sur le sujet. Avec environ 700 participants, des Assises nationales ont conclu en quatre jours de recommandations ce moment de "consultation du pays" sur une préoccupation qui en touche toutes les couches et régions (CNO-EGF, 2009). La formule est reprise du modèle de préparation du découpage communal dans les années 1990, avec ses débats organisés sur tout le territoire; après l'élection du président Ibrahim Boubacar Keita, elle inspirera d'autres " assises nationales" pour faire de même un état des lieux et des attentes sur la décentralisation (octobre 2013) puis sur le Septentrion malien (novembre 2013).

Selon ces deux angles, la désignation des problèmes fonciers oscille entre un vocabulaire technique sur le manque de cadastre, rappelé régulièrement depuis les années 2000 (Traoré, 2010), et le paradigme de la prédation :

"Aujourd'hui, des prédateurs fonciers, avec l'aide de leurs complices tapis dans l'administration, ont décidé de dépouiller riches et pauvres de leurs terres. Ils brisent des foyers et des vies... Après les États Généraux sur le foncier, le moment est venu de 
sévir" (intervention du ministre de la Justice, à l'occasion de la conférence des Parquets, octobre 2012).

Les deux années de crise qui ont suivi le putsch militaire de mars 2012 ont vu en effet ce lexique tenir lieu de compréhension de la relation de l'État à ses mondes sociaux. Même s'il n'est pas nouveau dans la région (Fay, 1995), le registre du " ventre " et son large bestiaire occupent toute prise de parole publique, du groupe de quartier à la colère institutionnelle du ministre ici cité, en passant par les exaspérations remontant de la rue et de blogs en ligne. À la fin de 2013, la restauration de l'autorité publique passe donc par un retournement du terme. Alors que la prédation désignait l'État avant la crise, certains de ses représentants s'emparent du terme pour déclarer l'État lui aussi "victime des prédateurs". Le nouveau président donne le ton dès son investiture en ciblant «la prédation des maigres ressources de l'État " (discours aux chefs d'État invités, 26 septembre 2013) :

"Il sera mis un coup d'arrêt à la magouille foncière et à la spoliation des pauvres ou des vrais titulaires. Tout sera fait pour doter le pays d'un système cadastral fiable et ce, dans des délais raisonnables. Il en sera fini des procès monnayés dans les bureaux des juges oublieux de l'éthique » (message à la Nation, 22 septembre 2013).

\section{Le scénario déjà éprouvé des " mesures d'urgence "}

$C^{\prime}$ est bien sur ce levier d'action du "parler droit " présidentiel ${ }^{2}$, que s'articulent en effet les termes désignant la perte d'autorité et les mesures de rupture visant à la rétablir. Les dispositions prises dès 2014, pour permettre au dossier de réforme de se monter, présentent pourtant la même ambiguïté à l'égard d'un passé proche.

L'arrêté $n^{\circ} 2014 / 2303$ du 21 août découle ainsi de trois ministères: Domaines de l'État, Intérieur et Sécurité, Décentralisation et Ville.

"[II] suspend les attributions de terrain du domaine immobilier de l'État et des collectivités territoriales pour six mois renouvelables, y compris les dossiers en cours d'instruction. "

${ }^{2}$ Le leadership d'Ibrahim Boubacar Keita s'incarne dans la formule kan kelen tigi désignant " celui qui n'a qu'une seule parole». 
Désignant des terrains ruraux et d'habitation, la mesure touche à pratiquement toutes les procédures; le dispositif interministériel semble aussi prouver une réelle coalition d'intérêts à la réforme. La suspension sera effectivement prolongée deux fois en 2015, jusqu'à sa levée complète à la fin de 2016.

Si la mesure permet d'organiser un réarchivage des dossiers papier, elle procède surtout d'une prévention habituelle à l'approche des élections locales $^{3}$ : il s'agit de détourner le Domaine national du risque d'instrumentalisation que l'échange promesse d'attribution foncière contre vote favorable a souvent démontré. Le procédé du coup d'arrêt administratif est loin d'être nouveau. II participe d'un bridage régulier des cessions domaniales, qui ne cesse en fait d'alterner avec des moments plus permissifs de recomposition des rapports clientélistes dans l'histoire contemporaine du Mali (Bertrand, 2006).

La Troisième République a connu ainsi plusieurs mesures de suspension des attributions foncières. C'est le cas entre 1996 et 1999, pendant une longue crise électorale, en 2003 et en 2006 à l'initiative toujours de l'Administration territoriale. Celle d'octobre 2011 se montre enfin interministérielle en impliquant l'Urbanisme, et en affectant la transformation de concessions rurales en titres fonciers définitifs. À l'approche des élections présidentielles prévues pour l'année suivante, elle désigne notamment les pratiques spéculatives de "promoteurs immobiliers » que la politique d'ouverture au privé du secteur du logement avait favorisés dans les années 2000. Elle illustre alors les tiraillements d'intérêts dans lesquels l'action publique se trouve en matière foncière.

Cette succession de mesures exceptionnelles prouve surtout la faible portée des dispositions réglementaires prises dans l'intervalle. Mais son lexique - "surseoir à toute attribution... jusqu'à nouvel ordre " - fait écho à celui de leaderships personnels qui se fondent sur le même langage de la fermeté : " moralisation des affaires publiques", " assainissement de la vie politique ", et finalement "le moment est venu de sévir... " du ministre de la Justice, durant l'intérim politique de 2012-2013. La posture

${ }^{3}$ Les scrutins régionaux et communaux sont d'ailleurs reportés à plusieurs reprises, du fait d'une insécurité persistante sur le territoire malien, et finalement dissociés. 
d'implacabilité du nouveau chef de l'État lui fait suite autour des mêmes désordres du foncier. Mais il s'agit désormais de fermer la parenthèse de la crise : le gouvernement enregistre la commande de réforme à son agenda.

\section{Le changement dans le recyclage}

Une fois les mesures d'urgence actées, le gouvernement doit surtout communiquer sur des "dossiers", non sur des " coupables ». Le montage financier de la réforme remet donc en selle la parole des experts :

"En fait, les constats établis lors des États Généraux du foncier, sur la gestion domaniale et foncière au Mali, sont toujours pertinents aujourd'hui : [...] manque à gagner pour l'État... faible incitation à l'investissement... conflits souvent très violents... dégradation du climat social... effets pervers sur le développement... confusion juridique... décisions contradictoires... perte de crédibilité... " (dossier de Réforme de la gestion foncière visant l'instauration d'un cadastre au Mali, octobre 2014 : 22-23).

L'ambiguïté sur le début de l'action s'inscrit là : il aura fallu en finir avec le système politique précédent pour dire ce que celui-ci disait déjà sur luimême. La multiplication des points géodésiques de rattachement des parcelles, l'appui aux collectivités territoriales en matière d'information foncière, l'informatisation du Livre foncier, le désengorgement d'une justice dont tous les recours convergent vers la Cour suprême : tout cela n'a pas dépassé le stade des incantations ou ne s'est pas traduit dans un agenda coordonné. Dans l'attente d'une levée de fonds significative, les nécessités d'action restent alors associées à un objet flou : le cadastre.

Les concurrences marchandes qui se déploient autour de la terre demandent pourtant une régulation; le lexique sécuritaire est mobilisé à la fois pour l'investissement économique et pour la cohésion sociale, ce qui débouche sur de prévisibles contradictions d'intérêts. Quelles seraient notamment les garanties données à des transactions foncières fondées sur d'autres droits qu'individuels et enregistrés ? Le cadastre n'est donc pas questionné sur son utilité sociale mais fait " assemblage " entre le courant des problèmes et le courant des solutions (Zittoun, 2013). Le terme n'est d'ailleurs pas bien défini sur un plan technique, ni dans les mots du président ni dans ceux du chef du gouvernement ouvrant la table ronde ; le lien qu'il semble établir entre les besoins et les résultats attendus n'est pas 
non plus clair (Ehwi et Asante, 2016). Le cadastre est présenté avant tout comme un bel objet informatique; il suscite une véritable invocation puisque sa "mise en œuvre effective" devrait valoir mobilisation politique, et faire oublier tous les maux. La formule dispense surtout de capitaliser les expériences antérieures, comme les enquêtes foncières avortées que le deuxième Projet urbain de la Banque mondiale avait lancées à Bamako dans les années 1980.

Le débat public évite ainsi de se demander pourquoi des mesures concrètes n'ont pas été prises après les recommandations de 2009, ni d'évaluer les outils faisant déjà succédané de cadastre depuis 2006 (Bertrand, 2018). Que ce soit avec la gestion communale des conflits, ou le projet d'observatoire foncier, le Mali est un cas typique d'amnésie institutionnelle. Mais devant les amis du pays, ses représentants donnent le cadastre à voir comme l'expression d'une large demande sociale, et en particulier celle des plaignants en justice dont les litiges - fonciers à $70 \%$, précise le Premier ministre - encombrent les tribunaux en désavouant les médiations traditionnelles. La compréhension de la "sécurisation foncière " n'est pas vraiment unifiée dans ces conflits (Djiré, 2006); mais I'instruction du cadastre continue de participer d'un rêve de consensus qui va au-delà du précédent mandat présidentiel. Dans l'immédiat, elle justifie de solliciter l'aide financière de l'Union européenne.

2014-2015 : trois gouvernements et deux styles ministériels dans l'an I de la réforme

Dans l'année de lancement du chantier, de plus fines lignes de positionnement politique s'expriment en interne autour de la commande présidentielle.

En 2014, le montage du dossier est confié au MDEAF et à ses partenaires européens: c'est sur lui, plutôt que sur le ministère des Finances, que reposent en effet les termes de référence et l'inventaire des dispositions à prendre. Quelques mois plus tard, la réforme prend le tour pénaliste d'un programme de traque des spéculateurs, avec retrait de titres « frauduleusement cédés par l'État » : c'est lui qui en 2015 donne au MDEAF une visibilité renouvelée dans l'opinion publique, et un crédit 
personnel à son représentant que l'on peut qualifier de force de réenchantement politique.

Le premier style met en jeu la capacité d'un service domanial discrédité à s'imposer face aux ministères poids lourds de l'État dans un cadre d'action inévitablement partagé sur la question foncière. Le second met en jeu une communication qui doit faire vibrer sur la durée la corde de la lutte contre la corruption.

De la réforme de l'action publique...

\begin{tabular}{|c|c|c|}
\hline $\begin{array}{c}\text { Chef du } \\
\text { gouvernement }\end{array}$ & Nomination-Départ & MDEAF \\
\hline $\begin{array}{l}\text { Oumar Tatam Ly } \\
\text { (sans étiquette } \\
\text { politique) }\end{array}$ & $\begin{array}{l}\text { Septembre } 2013 \text { - } \\
\text { avril } 2014 \text { : démission }\end{array}$ & \multirow[t]{2}{*}{$\begin{array}{l}\text { Tieman Hubert Coulibaly } \\
\text { (Union pour la démocratie } \\
\text { et le développement) }\end{array}$} \\
\hline $\begin{array}{c}\text { Mousa Mara } \\
\text { (parti Yéléma, alors } \\
\text { soutien présidentiel) }\end{array}$ & $\begin{array}{l}\text { Avril } 2014 \text { - } \\
\text { janvier } 2015 \text { : démission }\end{array}$ & \\
\hline $\begin{array}{l}\text { Modibo Keita } \\
\text { (ministre sous la } \\
\text { Ile République, } \\
\text { brièvement Premier } \\
\text { ministre en 2002) }\end{array}$ & $\begin{array}{l}\text { Janvier } 2015 \text { - } \\
\text { avril } 2017 \text { : démission } \\
\text { Remaniements } \\
\text { ministériels : } \\
\text { - septembre } 2015 \\
\text { - janvier } 2016 \\
\text { - juillet } 2016\end{array}$ & \multirow[b]{2}{*}{$\begin{array}{c}\text { Confirmé dans un ministère } \\
\text { fusionné Habitat, Urbanisme } \\
\text { et Affaires foncières }\end{array}$} \\
\hline $\begin{array}{l}\text { Abdoulaye Idrissa } \\
\text { Maïga (société civile, } \\
\text { ministre depuis 2014) }\end{array}$ & $\begin{array}{c}\text { Avril } 2017 \text { - } \\
\text { décembre } 2017: \\
\text { démission }\end{array}$ & \\
\hline $\begin{array}{l}\text { Soumeylou Boubèye } \\
\text { Maïga (ministre en } \\
2011 \text { et 2013) }\end{array}$ & $\begin{array}{l}\text { Janvier } 2018 \text { - } \\
\text { septembre } 2018 \text { : fin du } \\
\text { premier mandat } \\
\text { présidentiel Ibrahima } \\
\text { Boubacar Keita }\end{array}$ & $\begin{array}{l}\text { Disparition du ministère des } \\
\text { Affaires foncières; la } \\
\text { direction nationale des } \\
\text { Domaines est rattachée au } \\
\text { ministère des Finances }\end{array}$ \\
\hline
\end{tabular}

Dès 2014, les proches du président, gagnants des élections législatives, montrent leur impatience face à des remaniements ministériels qui ne leur cèdent pas suffisamment de portefeuilles face à des personnalités sans étiquette politique, des compétences reconnues sous les présidences antérieures ou forgées durant l'intérim de crise. Trois gouvernements se 
succèdent dans l'année, ce qui modifie assez vite la tête du MDEAF et fait craindre le risque d'une instabilité ministérielle. Avec les incertitudes qui pèsent sur la paix au Nord, le retour des logiques partisanes est cependant contrarié. Le redressement institutionnel se trouve même incarné, successivement, par deux personnalités qui ne sont pas d'emblée satisfaites de la responsabilité qui leur est confiée aux Domaines.

La première personnalité, T.H. Coulibaly, est connue pour un soutien pragmatique à la présidence des années 2000, mais il n'apparaît pas comme un spécialiste du dossier foncier. C'est plutôt son aisance dans la coopération internationale qui le montre en capacité de faire bouger les choses sur un problème domestique qui l'intéresse moins que les relations extérieures ${ }^{4}$. C'est durant son passage au MDEAF que la table ronde place l'ambassadeur de France comme chef de file des 24 PTF invités, et que l'ambassadeur de la délégation de l'Union européenne au Mali le précède dans la liste. Après l'intervention militaire de la France au Nord-Mali, la conférence de Bruxelles dédiée au renouveau du Mali (mai 2013) a déjà promis une aide financière de 3,285 milliards d'euros pour appuyer les politiques de réforme du pays. II s'agit ainsi de pousser le dossier du cadastre dans le Plan de relance et de développement durable esquissé par cette conférence de 56 donateurs.

En effet, la réforme met en avant un pilotage interministériel inédit, qui rassure à l'extérieur. Ces nécessités de coordination entre départements d'État ne sont pas seulement liées à la suspension administrative. Le service domanial est présenté comme le troisième poste de recettes publiques; sa contribution au budget national engage le ministère des Finances. De fait, la coopération canadienne assiste déjà la direction générale des Impôts pour la mobilisation des ressources intérieures du Mali et commence à faire scanner les pièces administratives de tous les titres fonciers.

Conçue par l'expertise étrangère, la chaîne technique de la sécurisation constitue bien un défi de taille pour une gestion des droits qui en est

\footnotetext{
${ }^{4}$ Ses attentes seront mieux satisfaites quand il prendra ensuite les charges de la Défense et des Anciens Combattants, de l'Administration territoriale, des Affaires étrangères et de la Coopération internationale.
} 
restée aux registres papier. Le chantier dépasse donc de beaucoup les dotations attribuées par ministère, et demande de plus de larges contributions institutionnelles. Ce rôle d'assemblage donné à la réforme heurte la pratique administrative, habituellement cloisonnée et hiérarchisée entre de " gros ministères "-Économie et Finances, Administration territoriale - et des compétences " techniques " plusieurs fois reformulées depuis les Travaux publics et l'Habitat. Mais il s'agit précisément de dépasser les relations à couteaux tirés et les concurrences financières qu'un développement taillé sur les " projets » des PTF n'a cessé de faire jouer depuis 30 ans.

Les rédacteurs du dossier de la réforme proposent ainsi de confier la présidence du comité interministériel de pilotage au représentant du MDEAF. II s'agit là de coordonner deux volets fonciers, l'un de stratégie politique, l'autre d'outillage technique. Or ce ministère est le plus petit, budgétairement, parmi les administrations invitées à rationaliser la gestion domaniale. Détaché d'autres tutelles administratives depuis 2000, il manque encore de bureaux dans certaines subdivisions régionales; les recettes qu'il génère transitent vers le Trésor par le biais de services de recouvrement dépendant des Finances; lequel aura, à travers le cadastre, la charge de recouvrer l'impôt foncier.

Le dossier chiffre enfin à 30 milliards de francs CFA - près de 46 millions d'euros - la mise en œuvre sur cinq ans des différentes activités. La participation financière recherchée à l'extérieur représente le double de celle attendue de l'État. Mais celle-ci est moins immédiate puisqu'attendue des recettes et d'économies que le pays espère retirer des effets vertueux du cadastre: un potentiel chiffré à 20 milliards de francs CFA par an à partir de 2019.

\section{sur le terrain \\ ... Au " ministre frondeur " : le crédit de la réforme déplacé}

En 2015, l'arrivée d'un nouveau responsable du MDEAF maintient l'ambition mais change le style. M.A. Bathily prend la charge des Domaines depuis le portefeuille Justice et Droits de l'homme qui lui a valu une stature et de nombreuses critiques dans le précédent gouvernement Ly. Le changement est commenté comme une disgrâce, car cet avocat de 
profession s'est plu à bousculer les magistrats. Département " technique ", le MDEAF est supposé le frustrer lui aussi.

D'emblée la communication du ministère est renforcée autour de sa personne, tandis que de nouveaux conseillers, venus avec lui de la Justice, l'orientent vers des sites sensibles. Des visites de terrain sont menées dans les alentours de la capitale et en régions pour lui permettre de répondre directement aux sollicitations qui, venues en nombre, l'embarquent sur le plaidoyer de justice sociale qu'il a déjà comme président d'un collectif d'associations. Avec un lexique de défense des démunis, le ministre se présente lui aussi " capable de dire la vérité " plutôt que de céder aux manœuvres visant à " décrocher n'importe quel siège ". C'est surtout "à la rencontre des citoyens", plus que sur un objet technique poussé de l'extérieur, qu'il forge sa légitimité aux Domaines : en se démarquant " de la vision rigide qu'ont du peuple les autorités dans notre pays et de la distance arrogante qu'ont prise les élites de Bamako à son égard » (entretien personnel, juin 2015).

Le positionnement politique de la réforme prend corps avec lui, qui multiplie les formules opposant "riches" et "pauvres " au lieu de les placer en égales victimes de la prédation. Ses prises de parole, en bambara autant qu'en français, réenchantent le débat de société qu'il faudrait selon lui oser aborder en profondeur, et une vision de la politique qui ne doit " rien céder aux politiciens".

Le ministre ne cesse alors de faire la une de la presse. Avec lui, "la gravité de la situation actuelle du foncier est passée au peigne fin!» (Tjikan, 9 juin 2015). La même année, il « accuse les autorités qui donnent les terrains sachant qu'elles n'ont pas le droit de les donner " ( $L a$ Révélation, 12 juillet); il " exhorte les Maliens à former un front contre l'accaparement des terres " (L'Indépendant, 17 juin). Ici il fait " paniquer » un élu ; là il " fait planer une épée de Damoclès » sur la tête d'un maire (Le Débat, 24 juillet). Ses délégations suscitent " liesse populaire » et tournent en "meeting de soutien "; il " défraie la chronique ", et "fait encore parler de lui " lors des séances parlementaires de questions au gouvernement. Pour les uns il joue au « justicier»; pour les autres il est « pyromane » de sa propre équipe (L'Aube, 27 juillet). 
La démarche se veut enfin pratique. Elle procède par retrait de ces titres fonciers "supposés fonder un droit irrévocable alors qu'ils sont attribués dans la confusion ". Une série de cessions administratives portant sur plusieurs centaines d'hectares déjà immatriculés à Bamako et dans le cercle voisin de Kati sont annulées ostensiblement. Et plutôt que de se laisser intimider par de "pseudo-opérateurs économiques" ou des " magistrats corrompus", il s'agit de "mettre en garde : chaque fois que ceux dont les titres sont annulés essayeront d'attaquer l'État, nous les attaquerons au pénal » (Le Prétoire, 8 juin 2015).

Un tel activisme médiatisé lui confère donc une posture de super ministre agissant vite et depuis le terrain, qui tranche avec son prédécesseur. C'est sur lui que reposent la réception sociale de la réforme et l'annonce de plus originales "mesures d'urgence »: définir des périmètres de sécurisation des terres agricoles, prévoir une riposte juridique aux spéculateurs qui se retourneraient contre l'État, suivre le cadastre sur la corde de la rectitude de l'État.

L'an I a donc fait ressortir deux forces de conviction, différentes l'une de l'autre, mais restant tributaires de leur ministère. Sur le fond, on peut se demander si la posture frondeuse de la seconde, à l'égard du gouvernement, ne relève pas d'une vision réaliste des contraintes politiques pesant sur la sortie de crise : la mise en place des accords pour la paix se montre difficile, et plusieurs attentats ont touché le sud du pays ; après deux gouvernements qui ont peu duré, des PTF hésitent à penser que l'action publique est réellement refondée. Ces contraintes conduisent le chef de l'État à bouger souvent les cases gouvernementales. Elles semblent conduire le patron des Domaines à des perspectives d'action courtes, voire au coup d'éclat. L'enjeu n'est pas nécessairement de rester sur ce chantier, dont l'évaluation se fera évidemment sur un temps long. II est plutôt d'avoir démontré une carrure politique, de ménager la possibilité de faire rebondir ailleurs ce " parler franc, agir droit ».

Le premier remaniement ministériel de 2015 lui donne raison: le ministre est reconduit, comme si oser la posture payait dans ce temps incertain. La force de conviction qu'il met à l'actif de l'État sur le registre de la justice sociale, bien qu'assez vague sur ses lignes d'adversité, semble jouer un rôle politique au moins à court terme. Faute de recette miracle 
pour transformer l'administration, les impatiences populaires seront contenues par de bons coups de communication sur la dilapidation des ressources de l'État. De quoi gagner aussi du temps avant les prochaines élections, pour un scrutin local qui mobilise peu la société malienne.

2015-2016 : un ministère cantonné sur le volet technique et en butte à l'afflux de demandes domaniales

Un an après la table ronde, le chantier foncier glisse déjà vers une mise en œuvre plus sectorielle que réellement transversale. Le MDEAF doit déjà aborder quelques désenchantements puisque les soutiens financiers attendus ne sont pas au rendez-vous. De plus, la bureaucratie domaniale se montre moins intéressée que son représentant par une appropriation de la réforme dans les termes de la justice sociale.

\section{L'Europe absente du financement, des feuilles de route distinctes au gouvernement}

La logique d'extraversion qui pèse sur l'État du Mali s'illustre d'abord dans le fait que le partenaire européen sollicité pour une aide budgétaire directe ne donne pas satisfaction sur le volet d'action foncier. Le chantier de la décentralisation, notamment au nord du pays, lui fait concurrence comme priorité à soutenir. La diplomatie française est de plus absorbée par l'Accord pour la paix et la réconciliation au Mali, finalement signé en juin 2015, dont l'application se montrera laborieuse. Corrélativement, l'approvisionnement gouvernemental du programme de réforme est lui aussi révisé à la baisse, avec 1 milliard de francs CFA - plus d'1,5 million d'euros - au lieu des 10 milliards attendus. C'est finalement la Banque mondiale qui retient la confection du plan cadastral de la capitale et la relecture du Code domanial et foncier comme indicateurs de son appui budgétaire global au Mali : des projets inscrits au budget de l'État pour la reprise économique et la réforme de sa gouvernance.

Le statut d'État faible qui colle au Mali, débordé par ses problèmes sécuritaires, soulève ainsi son lot d'inquiétudes et retarde l'approbation du cadre institutionnel de la réforme foncière. Elle ne viendra, par décret présidentiel de mars 2016, qu'en modifiant sensiblement le format prévu 
initialement par le MDEAF : la présidence du comité d'orientation de la réforme revient au Premier ministre et non au ministre porteur du dossier. Celui-ci se retrouve en position subordonnée avec la présidence d'un comité technique de pilotage annonçant huit groupes sectoriels et un Secrétariat permanent de la réforme domaniale et foncière. Au gouvernement revient donc la responsabilité du document de Politique nationale domaniale foncière, référence unique pour toutes les politiques sectorielles relatives à la question. Quant au plan d'action sur la sécurisation des droits fonciers, il est redessiné par le MDEAF à l'horizon 2020-2021 : son coût total est porté au double des prévisions de 2014, soit plus de 60 milliards de francs CFA, mais la part de l'État malien monte aux deux tiers, ce qui maintient la demande de 20 milliards aux PTF.

Chapeautée par le chef du gouvernement, la réforme voit donc sa dimension interministérielle reconnue, mais elle ne laisse pas de rôle de coordination stratégique au ministère des Domaines. C'est sans doute aussi la résultante d'un calcul d'équilibre dans lequel le MDEAF reste perçu comme "petit et technique ". Les titres définitifs qu'il gère occupent certes le haut du dispositif de formalisation des droits de propriété, mais ils ne représentent qu'une partie des attentes sociales. Ils ont certes un potentiel fiscal fort, mais placent le département dans une relation asymétrique avec celui des Finances, auquel revient la mission de recouvrement des recettes de l'État.

La territorialité du service domanial apparaît de même encore faible par rapport à celle de l'Administration territoriale, car les services déconcentrés et les collectivités décentralisées qui ont cette dernière pour tutelle disposent d'archives et de commissions domaniales propres. La décentralisation et le dernier Code foncier de 2000 ont en effet démultiplié les guichets d'attribution de concessions rurales ou d'habitation dans les régions, les cercles, les arrondissements et les communes. Ces droits provisoires entretiennent la confusion avec les droits définitifs, plus sélectifs, d'autant que le maillage politico-administratif qui les fonde est au contact direct des autorités traditionnelles, et porte l'essentiel de la relation gouvernants/gouvernés en matière foncière. La réforme cherche alors à obliger ce ministère des Affaires intérieures à renoncer à l' « excès d'autorités " qui le caractérise en matière de concessions foncières. Mais la feuille de route de ce département d'État est pour l'heure ailleurs : dans 
le fait de raccrocher au plus vite les communautés et le développement économique du Septentrion au reste du Mali.

Enfin, le ministère du Développement rural ressort la même année en phare d'une autre mobilisation touchant aux ressources naturelles alors qu'il ne figurait pas dans le premier cercle d'intéressement à la réforme. II est rappelé au gouvernement qu'un projet de loi foncière agricole est annoncé depuis déjà dix ans dans le document assez complet voté par les députés en 2006; cette Loi d'orientation agricole a pris acte de la reconnaissance juridique des droits coutumiers et annonce, pour les enregistrer, la mise en place de commissions foncières dans les communes rurales. Le plaidoyer paysan qui fait remonter l'attente est moins intempestif que celui du ministre des Domaines, mais il est structuré au plan syndical. Divers activistes et organisations de la société civile lui donnent un écho international; la Convergence malienne contre l'accaparement des terres multiplie notamment ses interventions depuis 2014.

La concrétisation de cette loi relative au foncier agricole reste certes chargée d'incertitudes. Mais sur ce plan aussi, le positionnement du représentant du MDEAF est "doublé " par des organisations mieux averties que lui des vulnérabilités et tensions que vit le monde rural. II ne resterait donc à son ministère qu'à protéger les espaces publics et les ceintures agricoles des villes. Or le terrain urbain et périurbain est aussi plus complexe qu'il n'y paraît en quelques mots de " refonte des rapports sociaux " mis en exergue par le nouveau Secrétariat permanent. Dans une carte foncière mitée par les propriétés immatriculées, avec une spéculation plus diffuse que ce qu'en disent les accusations de fraude lancées par le ministre, la définition de périmètres d'aménagement et de ravitaillement alimentaire revient vite à vouloir gérer l'ingérable.

\section{L'ordinaire des bureaux domaniaux}

La principale limite de l'impulsion ministérielle se joue en effet dans le quotidien du service domanial, qui manque cruellement de ressources humaines, matérielles et numériques pour traiter à la base une demande d'immatriculation exponentielle. Le problème ne se résume pas à l'inertie d'une culture bureaucratique rivée à ses procédures ; il relève également 
de ses normes pratiques travaillées par la corruption; il relève enfin du hiatus entre ces moyens du bord et une injonction constante à réformer la gouvernance africaine dans un empilement de projets et de rappels réglementaires (Bierschenk et Olivier de Sardan, 2014).

Dès avant le tournant démocratique des années 1990, la gestion foncière des collectivités locales se montre en effet multi-située entre des services déconcentrés, diverses médiations sociales et déjà quelques commissions communales. Pour la petite bureaucratie domaniale, l'annonce de la réforme en 2014 vaut promesses d'étagères et de groupes électrogènes; mais il ne s'agit pas de renoncer à répondre aux sollicitations personnalisées et aux demandes d'accélération dont elle est déjà la scène: que ce soit pour purger des droits de jouissance antérieurs au bornage d'une parcelle; pour évaluer la mise en valeur requise d'une concession; pour attribuer des lots à certains en délogeant d'autres usagers; pour déroger même au parcours du combattant que représente la transformation de droits provisoires en titres définitifs de propriété.

Dans cet État banalement à l'œuvre, le chef du bureau des Domaines et du Cadastre du cercle de Kati estime alors qu'il « ne fait pas le poids » face au préfet et son service domanial propre (entretien personnel, février 2015) : a fortiori depuis que celui-ci, au tournant des années 2010, devient l'épicentre d'un réseau de démarcheurs des terres coutumières à mobiliser aux portes de la capitale pour les revendre aux citadins sous la forme de lots à bâtir. Si le préfet n'a plus la compétence d'attribuer des parcelles d'habitation, ses "bulletins" - petits papiers - et ses "coxers" rabatteurs de clients - s'en chargent, au grand dam de maires doublés dans leur prérogative communale, et surtout dans leurs propres pratiques officieuses.

L'autorité administrative reste donc structurante dans ce dispositif local, social autant qu'institutionnel. Les services d'ingénierie - Génie rural et Élevage, Hydraulique et Urbanisme - restent comme les Domaines perçus comme "techniques", c'est-à-dire subordonnés au pouvoir discrétionnaire du préfet ou du gouverneur de région. Pour le requérant de base, toutes ces verticalités se traduisent en nécessités à négocier : avec la publicité à donner aux procédures de bornage contradictoire, avec les seuils de compétence territoriale définis selon la superficie des terrains 
convoités, avec la vocation des sols, avec les mutations de droits pour règlement d'héritage, etc.

La routine domaniale se trouve pourtant bousculée par un afflux exceptionnel de demandes de titres. Entre 2006 et 2014, le Livre foncier malien a explosé en passant de 73000 à 188000 enregistrements, sans que sa direction nationale ne produise aucun bilan des régions et des bureaux, dont les six derniers installés en 2014 et 2015. Dans cette dynamique, le cercle de Kati concentre à lui seul près de $60 \%$ des titres enregistrés au Mali, et ceux de 2014 en disent long sur la dérégulation des procédures de cession administrative : pas moins de 22000 titres sont délivrés dans l'année; bien au-delà du précédent pic d'avant la crise: 9000 titres en 2011. Le flux se confirme en 2015 au point que le stock de droits formels double en seulement trois ans, de 60000 à près de 120000 titres créés en périphérie de la capitale.

La suspension décrétée d'en haut n'a donc pas d'effet autre que pervers, et montre la force d'arrangement de la demande avec la bureaucratie locale. Le coup d'arrêt donné à la transformation des concessions en titres stimule ainsi d'autres stratégies d'accumulation foncière: il favorise d'abord les passe-droits qui sont déjà rodés à des " cessions directes " depuis les années 2000 ; surtout, il reporte la création de titres sur des manœuvres privées et sur de petites parcelles de $300 \mathrm{~m}^{2}$. Ces lots destinés à l'habitation sont issus du morcellement de titres-mères que l'État a déjà enregistrés; les droits liés à l'immatriculation sont simplement démultipliés par un jeu d'écriture qui peut garantir des plusvalues à bon compte à leurs titulaires: la propriété source est cédée administrativement à 80 francs CFA le mètre carré, et parfois à la moitié du prix réglementaire du fait de faveurs tarifaires; mais les lots, chacun doté d'un nouveau titre, sont revendus entre 5000 et 6000 francs CFA le mètre carré, sans guère de viabilisation. Cette filière marchande échappe ainsi à la suspension administrative et représente 80 \% des titres créés en 2014. De fait, elle n'a cessé de se développer depuis que le montage de ces dossiers de "lotissement" revient à des géomètres privés ${ }^{5}$. Une fois

${ }^{5}$ Leurs prestations sont souvent rémunérées en nature. En dix ans, ce groupe professionnel a gagné une forte visibilité parmi les détenteurs de titres. 
libéralisée, la profession continue de suivre le circuit d'autorisation par le service domanial et celui de validation technique par d'autres directions régionales, désormais pour le compte de clients particuliers, principalement des commerçants. Bon nombre de notaires encouragent également les détenteurs de titres à mobiliser en hypothèques, auprès des banques, ces portefeuilles fonciers qui leur valent des facilités d'accès au crédit commercial.

Les entreprises privées de parcellement ne suscitent pourtant guère d'investissement immobilier; elles perturbent au contraire l'opérationnalisation de décisions d'aménagement urbain et condamnent à l'avance les places publiques qui n'y sont programmées que sur le papier. Leur visa technique est donné sur la seule mention " réalisé à $100 \%$ » concernant le bornage, tandis que les rubriques de voirie-réseaux divers en restent à $0 \%$. L'appel à la fermeté et au service de la Nation lancé par le ministre, la remise en ordre des dossiers fonciers attendue de la suspension, sont donc loin d'enrayer cette dynamique de titrisation déjà débridée par des sousproduits d'immatriculation. Ni 2015 ni les années suivantes ne verront la reconstitution des réserves foncières que l'État a démantelées pour le compte d'opérateurs économiques incertains; et pas davantage la mise en défens de zones agricoles autour de la capitale.

\section{6-2017 : l'horizon électoral de l'action publique}

Avec la mise en place d'une nouvelle structure institutionnelle, le Secrétariat permanent à la réforme, celle-ci s'achemine à sa troisième année vers une obligation de résultats. Les élections présidentielles de 2018 se profilent à l'horizon, la commande de cadastre aura donc besoin d'être rappelée à l'actif du mandat en cours et comme argument d'une conduite des affaires publiques meilleure qu'avant la crise.

\section{Risques d'usure du leadership ministériel}

Si le style Bathily a fait mouche en 2015, sa performance politique mérite d'abord d'être questionnée sur la durée : d'une part du fait de la longueur de la mesure de suspension administrative; d'autre part comme entrepreneur de normes de révision en profondeur de la gestion foncière. 
Ses décisions d'annuler des titres sont elles-mêmes annulées par la Cour suprême du Mali, qui donne raison aux titulaires de ces droits formels de propriété.

Sur le fond, la posture du ministre est héroïsante, plus individuelle qu'institutionnelle: il est sollicité comme sauveur providentiel de communautés déchirées par la marchandisation de leurs terres et par l'appétit de nantis. Plus qu'à des critères d'évaluation du travail collectif, ce positionnement du " juste défenseur » en appelle à de la reconnaissance politique, qu'il situe tantôt du côté du peuple, tantôt du côté du président en faisant fi du chef du gouvernement. C'est comme cela qu'il faut comprendre la longévité d'un "ministre frondeur " à un poste sensible: entre fidélité affichée au chef de l'État, contre les membres de son parti, et instrumentalisation par celui-ci du rôle de pompier que tient celui-là face au risque toujours fort d'embrasement populaire dans les affaires foncières.

La posture se montre d'autre part peu portée à poser des actes concrets de renforcement des capacités administratives sur le terrain, y compris de formation et d'équipement que demande le personnel. Les visites de quartier laissent les tensions non dissoutes; des opinions adverses à celle du ministre, lésées dans leur intérêt par ses propos ou ses annulations, se manifestent; d'autres ministres craignent qu'il aille trop loin en bousculant les élus, parmi lesquels le fils du président, ou en tirant une balle dans son propre ministère.

Enfin, la posture n'échapperait pas, elle non plus, au risque de compromission personnelle au fur et à mesure que le blocage des demandes de titres se prolonge. Des détracteurs, des déçus, des concurrents de la société civile commencent à "révéler des affaires "; le traitement dérogatoire de demandes d'immatriculation grossit un sentiment d'injustice et le soupçon de favoritisme, mêlant attaques personnelles et discrédit plus général " des hommes corrompus par le pouvoir ". La suspension administrative devient contre-productive au sein du MDEAF : elle créé un bouchon d'attentes qu'il faudra bien faire sauter comme le souhaitent des responsables du service dans des régions où l'État est en réelle déshérence depuis 2012. Il devient de même évident qu'en bloquant les affectations domaniales aux communes, au lieu de les 
faire traîner comme d'habitude, on justifie leurs pratiques informelles de lotissement.

Les visites de terrain s'espacent donc en 2016, quand de nouvelles techniques et un impératif d'aller désormais vite prennent le relais des discours sur la réforme.

"Les propriétaires sont invités à matérialiser les limites de leurs parcelles, bâties ou non bâties, à usage d'habitation ou de champ ॥

La relecture des textes fonciers se veut d'abord diligente :

"Les experts du groupe sectoriel et les consultants qui ont rendu leur rapport, c'étaient tous des nationaux; on a pu tout boucler en 2017: l'avant-projet de Code domanial et foncier et le tableau comparatif des changements avec le code actuel " (entretien avec le secrétaire, octobre 2017).

Mais il s'agit surtout de rendre la réforme concrète en communiquant sur son action prioritaire : confectionner le Cadastre dans le district de Bamako et le cercle de Kati. C'est en effet ce train de dispositions techniques - attribution à chaque parcelle d'un numéro d'identification nationale, lancement des enquêtes foncières et fiscales, installation d'une application Web pour " permettre aux citoyens d'être informés par SMS téléphonique de l'enregistrement de leurs biens dans les bases de données cadastrales »-qui est censé outiller sur la durée la réforme de la fiscalité locale et la gestion des conflits fonciers. Les enquêtes annoncées pour la mi-2017 devront cependant attendre la fin des levés géographiques. Elles se contenteront de ressources budgétaires limitées en 2018.

Avec le glissement de l'insécurité militaire du nord au centre du pays, on perçoit plus que jamais la contraction de l'État " actif " sur ce qui est désormais appelé le sud du pays. Les levés de coordonnées géographiques sont lancés officiellement en novembre 2016. Les communes concernées, six à Bamako puis sept à ses bordures, un an plus tard, soulignent la surambition donnée à cet inventaire de parcelles urbaines et périurbaines : " refonder les rapports entre les populations et l'administration ». Mais le géoréférencement est bien inédit à cette échelle; il crée un effet d'aubaine pour les géomètres-experts qui se voient confier un matériel 
GPS de haute précision et la sous-traitance de l'opération pour le compte du Secrétariat permanent. À son équipe... de quatre personnes, dont un seul ingénieur, incombe la supervision de la base de données créée pour la circonstance.

On sort alors d'une logique de reconnaissance des seuls titres fonciers. Toutes les parcelles de Bamako sont ainsi recalées en 2017 à partir des plans existants et de nombreux correctifs de terrain : il faut tenir compte des biens subdivisés par les familles et de nombreux lots créés à la discrétion des édiles. Mais l'écart entre plans et réalité ne fait l'objet d'aucun bilan chiffré. II est surtout demandé aux propriétaires de matérialiser leurs limites par le scellé de bornes ou l'introduction de matériaux de clôture visibles dans les voisinages. Les levés reviennent donc à valider numériquement toute parcelle inscrite physiquement au sol et à entériner les positions de force dont ces limites du jour procèdent souvent.

L'obligation d'aller vite en opérations colle en effet à la perception sociale des enjeux fonciers: celle d'une course de vitesse qui consacre la légitimité du plus réactif aux annonces, et qui fait gagner l'investisseur contre celui qui traîne ou tergiverse. Face à des collectifs d'héritiers qui se montrent souvent divisés dans les vieux quartiers, face à la demande de parcelles à bâtir en périphérie d'agglomération, la communication sur le cadastre pourrait encourager certains à consolider leurs clôtures et d'autres à précipiter des "réalisations" destinées à faire valider un existant jusque dans ses formes insoutenables : des parcelles créées sur les dépôts d'ordures que sont devenues certaines places publiques avant d'être mises en pièces et vendues par les élus locaux; des bâtiments implantés sur des voies de passage ou le lit de marigots; des bornes discutables après double attribution du même terrain; etc. On peut craindre ici que soient de nouveau favorisés ceux qui savent déjà aller vite en clôtures comme ils l'ont fait en procédures :

"On ne pourra jamais aller en justice pour tous les problèmes d'empiètement, il faut fixer les limites, c'est comme ça qu'on fera avancer les choses " (entretien avec le secrétaire, ibid.).

Sous l'obligation d'efficacité se profile donc un détournement de sens sur la sécurisation, dont la gestion foncière a montré plus d'une fois les risques dans l'histoire du pays. 
À la fin de 2017, ce bilan quantitatif des plans cadastraux - plus de 200000 parcelles numérisées dans la capitale - répond donc à la commande présidentielle en montrant sur le fond l'absence persistante d'accompagnement social des politiques maliennes. Avec ses actes de classement et de communication, la carte a fait "raison graphique » (Goody, 1979) et a donné un coup d'accélérateur à l'action publique. Mais elle place en porte-à-faux un ministre qui a vu dans la réforme un levier pour rétablir les citoyens dans leurs droits mais n'a pu infléchir la réalité du terrain : celle des convoitises marchandes et des épreuves de force qui déferlent sur des communes mal outillées en durcissant les rapports sociaux et en laissant sur la touche les plus vulnérables des ayants droit communautaires.

\section{Conclusion}

La démission du Premier ministre qui intervient en décembre 2017 tourne une page en étant suivie de celle du ministre des Domaines. Ces remplacements, à quelques mois des élections présidentielles puis au début du mandat suivant, sont également marqués par la disparition des Affaires foncières dans l'organigramme gouvernemental.

En quelques années, trois niveaux d'action - format institutionnel, style ministériel, ordinaire bureaucratique - auront donc balisé les ambitions et les tiraillements de la réforme foncière malienne, complétant une trajectoire déjà chargée en incertitudes, oublis d'expériences, réenchantements de l'intérêt général, et vision morale de la spéculation foncière.

Associée en 2014 à la réforme de l'action publique, celle de la gestion foncière reste intimement liée à un ressenti de crise : la dégradation des conditions d'accès à la terre a plongé le pays dans un doute sur l'État et ses représentants ; mais la perte d'intégrité et de sécurité territoriales retarde ou contracte le sursaut de réponses publiques. De même évoluent les formes de l'extraversion pesant sur ces réponses: du gavage d'aides et de conditionnalités au développement à une présence militaire étrangère qui se pérennise. 
Depuis la capitale, cependant, le courant de remise en ordre des affaires publiques et celui des problèmes se sont retrouvés autour de la référence au cadastre. Si l'outil relève bien de pressions internationales, le fantasme de sécurité individuelle, le rêve de modernité numérique et l'imaginaire d'une gouvernance débarrassée de la corruption ont également progressé dans la société malienne. À défaut de "régler tous les maux", les levés de limites parcellaires ont stimulé une ingénierie urbaine et rallié de nombreux propriétaires à la course aux titres. Ils relancent le jugement politique local en montrant qu'une autorité fait enfin quelque chose, quand le précédent régime était resté en panne d'action.

Introduit par la campagne présidentielle de 2013, revigoré par les diatribes ministérielles, retraduit en termes policés par le Secrétariat permanent, ce processus discursif "des solutions au défi foncier " pose en effet quelques jalons techniques. II relie surtout des enjeux vite contradictoires dans le débat de société : ressources fiscales et prévention des conflits, sécurité des investisseurs et respect des droits coutumiers. Assurément l'administration cherche à se débarrasser de l'étiquette " complice de la prédation » qui lui colle sur toute une chaîne de services de l'État.

$\mathrm{Au}$ quotidien, la gestion foncière est pourtant moins enchantée et démontre par la base la difficulté de l'État à réguler ses échanges politiques avec la société malienne. Une nouvelle réforme, celle de la gouvernance locale, devra donc confirmer l'essai : dans les communes rurales, au sein desquelles des commissions foncières sont à mettre en place; au sein des bureaux domaniaux, qui restent dans l'obligation d'approvisionner les caisses de l'État; dans les nouvelles régions, en charge de stratégies économiques mieux concertées.

\section{Bibliographie}

BERTRAND M., 2006, "Foncier débridé / foncier bloqué. Enjeu récent de la décentralisation ou alternance centrale dans l'histoire longue des communes urbaines maliennes ? ", in FAY C., KONÉ Y.F. et QUIMINAL C. 
(dir.), Décentralisation et pouvoirs en Afrique. En contrepoint, modèles territoriaux français, Paris, IRD : 179-198.

BERTRAND M., 2014, "Mobilisations foncières à Bamako: des défis de la gouvernance à ceux de la citoyenneté ", in BRUNET-JAILLY J., CHARMES J. et KONATÉ D. (éds), Le Mali contemporain..., Bamako, Éditions Tombouctou et IRD : 159-186.

BERTRAND M., 2019, "A Cadastre for Mali? The production of land titles and the challenge of property data on the periphery of Bamako ", Land Use Policy, 81 : 371-381.

BIERSCHENK T. et OLIVIER DE SARDAN J.-P. (éds), 2014, States at work. Dynamics of African Bureaucracies, Leiden, Brill, Africa-Europe Group for Interdisciplinary Studies.

BRUNET-JAILLY J., CHARMES J. et KONATÉ D. (dir.), 2014, Le Mali contemporain, Bamako, Éditions Tombouctou et IRD.

COMMISSION NATIONALE D'ORGANISATION DES ÉTATS GÉNÉRAUX DU FONCIER (CNO-EGF), 2009, "Assises des États Généraux du Foncier » et "Annexes ", Bamako, ministère du Logement, des Affaires foncières et de l'Urbanisme, $159 \mathrm{p}$.

DARBON D., 2002, "La Culture administrative en Afriques: la construction historique des significations du "phénomène bureaucratique" ", Cadernos de Estudos Africanos, 3 : 65-92.

DJIRÉ M., 2006, « Immatriculation et appropriation foncière dans une zone périurbaine du Mali. Les avatars d'une procédure (nécessaire ?) ", in "Colloque international: Les frontières de la question foncière. Enchâssement social des droits et politiques publiques ", Montpellier, 1719 mai 2006, https://www.mpl.ird.fr/colloque_foncier/Communications/ PDF/Djire.pdf (consulté le 5 février 2019).

EHWI R.J. et ASANTE L.A., 2016, "Ex-Post Analysis of Land Title Registration in Ghana Since 2008 Merger. Accra Lands Commission in Perspective ", SAGE Open, April-June 2016 : 1-17, DOI : 10.1177/2158244016643351.

FAY C., 1995, "La démocratie au Mali, ou le pouvoir en pâture ", Cahiers d'études africaines, $137: 19-53$.

GOODY J. (trad.), 1979, La Raison graphique. La domestication de la pensée sauvage, Paris, Les Éditions de Minuit. 
KONATÉ D. (dir.), 2013, Le Mali entre doutes et espoirs. Réflexions sur la Nation à l'épreuve de la crise du Nord, Bamako, Éditions Tombouctou.

SOW B.-A.-S., 2016, Droits fonciers urbains au Mali. De son évaluation à sa réforme, propositions pour une meilleure sécurisation des transactions foncières, Brinon-sur-Sauldre, Éditions Grandvaux.

TRAORÉ M., 2010, « Les outils de la sécurisation foncière au Mali, conférence au séminaire de sensibilisation à la sécurité foncière à l'intention des parlementaires d'Afrique francophone ", 14-15 avril 2011, Bamako, Assemblée nationale de la République du Mali, http://apf.francophonie. org/Les-outils-de-la-securisation.html (consulté le 5 février 2019).

ZITTOUN P., 2013, La fabrique politique des politiques publiques, Paris, Les Presses de SciencesPo.

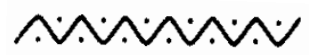

Monique Bertrand est directrice de recherche à l'IRD, membre du CESSMA

CESSMA-UMR 245 IRD, université Paris Diderot (France)

E-mail : monique.bertrand@ird.fr 